
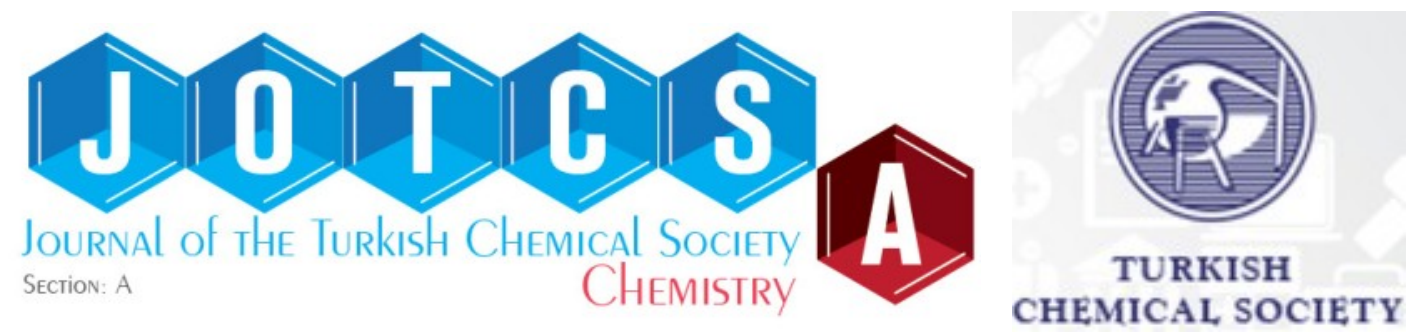

\title{
Quantitative Structure-Activity Relationship (QSAR) Studies and Molecular docking Simulation of Norepinephrine Transporter (NET) Inhibitors as Anti-psychotic Therapeutic Agents
}

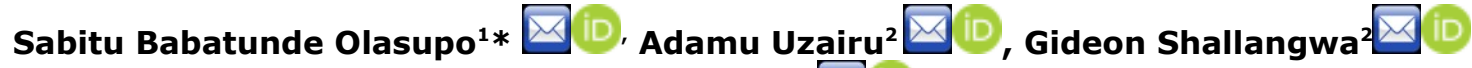 \\ and Sani Uba $\triangle$ \\ ${ }^{1}$ National Agency for Food and Drug Administration and Control (NAFDAC), Nigeria. \\ ${ }^{2}$ Department of chemistry, Ahmadu Bello University Zaria, Nigeria .
}

Abstract: The Norepinephrine transporter (NET) is a $\mathrm{Na}^{+} / \mathrm{Cl}^{-}$coupled neurotransmitter transporter responsible for reuptake of released norepinephrine (NE) into neural terminals in the brain, an important therapeutic agent used in the treatment of psychiatric disorders. A quantitative structural activity relationship (QSAR) investigation was carried out on 50 Molecules of NET Inhibitors to investigate their inhibitory potencies against norepinephrine transporter as novel agents for antipsychotic disorders. The molecules were optimized by employing Density functional theory (DFT) with basis set of $B_{3} L Y P / 6-31 G^{*}$. The genetic function Algorithm (GFA) approach was used to generate a highly predictive and statistically significant model with good correlation coefficient $R^{2}$ Train $=0.952$, Cross validated coefficient $\mathrm{Q}^{2}{ }_{\mathrm{cv}}=0.870$ and adjusted squared correlation coefficient $\mathrm{R}^{2}$ adj $=0.898$. The predictability and accuracy of the developed model was evaluated through external validation using test set molecules, Y-randomization and applicability domain techniques. The results of Molecular docking simulation by using two neurotransmitter transporters PDB ID 2 A65 (resolution $=1.65 \AA$ ) and PDB ID 4M48 (resolution $=2.955 \AA$ ) showed that two of the ligands (compound numbers 12 and 44) having higher binding affinity were observed to inhibit the targets by forming hydrogen bonds and hydrophobic interactions with amino acids of the two receptors respectively. The results of this study are envisaged to provide very important new insights into the molecular basis and structural requirements that would help in designing more potent and more specific therapeutic anti-psychotic agents.

Keywords: Norepinephrine transporter Inhibitors, antipsychotic, QSAR, Disorder, DFT, Drug.

Submitted: June 13, 2019. Accepted: November 26, 2019.

Cite this: Olasupo SB, Uzairu A, Shallangwa G, Uba S. Quantitative Structure-Activity Relationship (QSAR) Studies and Molecular docking Simulation of Norepinephrine Transporter (NET) Inhibitors as Anti-psychotic Therapeutic Agents. JOTCSA. 2020;7(1):179-96.

DOI: https://doi.org/10.18596/jotcsa.577259.

*Corresponding author. E-mail: olasabit@yahoo.com. 


\section{INTRODUCTION}

Mental disorder or psychotic disorder is a clinical syndrome in which some loss of contact with reality has occurred and it is generally applied to persons whose mental functioning is sufficiently impaired to interfere with their capacity to meet the ordinary demand of life (1). Psychotic disorders are common to all countries and cause immense human suffering, social exclusion, disability, poor quality of life, staggering economic and social costs. It is estimated that one in every four people have a mental disorder(1). The combined costs of mental disorder, including loss of productivity, loss of earning due to illness and social costs, are estimated to total at least USD 113 billion annually (2). The major depressive disorders (MDDs) had been estimated as the second largest global burden among all diseases by 2030 which makes the discovery of novel and efficacious anti-psychotic drugs very urgent (3). Persons with psychotic disorder are at risk for complications and derivatives' effects of psychosis such as suicide attempts, substance abuse, homelessness, victimization by others and committing act of violence (4).

Norepinephrine (NE) is a neurotransmitter, a crucial neurochemical messenger employed in central noradrenergic and peripheral sympathetic synapses (5) responsible for reuptake of released norepinephrine (NE) into nerve terminals in the brain. Dysregulation of this neurotransmitter is associated with many debilitating psychotic disorders and mental illnesses (6). Inhibition of the norepinephrine transporter by NET inhibitors has emerged as important drug targets with a multitude of therapeutic potentials for the treatment of psychiatric disorders and mental diseases (7).

Quantitative structure-activity relationship (QSAR) analysis is a useful technique to find correlations between biological activities and molecular descriptors of different classes of compound (8). QSAR plays a significant role in novel drug discovery, and it finds application in predicting the activity of novel compounds by mathematical expression which figure out the relationships between a chemical structure to their biological activity and a QSAR models give information that is very useful for drug design and medicinal chemistry.
In recent time, computer assisted drug design base on QSAR has been of great important to develop novel medications for the treatment of different ailments (9).

The aim of this study is to build up a QSAR model to explore the inhibitory potency of some NET inhibitors and likewise to elucidate the interactions between the inhibitor compounds, and the receptor sites.

\section{MATERIALS AND METHODS}

\section{Dataset collection and Geometry optimization}

A dataset of fifty (50) compounds of norepinephrine transporter (NET) inhibitors were sourced from CHEMBL Database.

Optimization is the process of finding the equilibrium or concept energy geometry of molecules. Chemdraw software Ultra-version 12.0 was used to draw the chemical structures of the compounds and subsequently imported into Spartan 14 software (10) to optimize the molecular geometry at the Density Functional Theory (DFT) using the $B_{3} L Y P$ at $6-31 G^{*}$ basis set (11) to generate quantum chemical and molecular descriptors.

\section{Division of Dataset}

The dataset of the studied compounds was partitioned into a training set and a test set by using Kennard stone algorithm (12) "Dataset Division GUI 1.2" software. The training set was used to develop the QSAR model, while the test set was employed to validate the developed model.

\section{Model Building}

A statistical analysis by genetic function approximation (GFA) techniques in the Material studio software 8.0 version was used to build the QSAR models. GFA has a distinctive attribute to generate a population of model equations rather than a singular model as most other statistical methods do. It also selects the basic function genetically, generate better models than those made using stepwise regression techniques. The range of variations in this population gives added information on the quality of fit and importance of the descriptors (13). The Friedman's Lack of Fit (LOF) was employed to evaluate the quality of the model as a method that measures fitness of a model. LOF is estimated by this mathematical expression; 


$$
L O F=\frac{S E E}{(1-(C+d \times p) / M)^{2}}
$$

Where $c$ is the number of basic functions, $d$ is the smoothing parameter, $M$ is the number of samples in the training set, SSE is the sum of square error and $\mathrm{p}$ is the sum number of descriptors contained in the model.

\section{Molecular descriptors calculation}

Molecular descriptors are arithmetical values that describe properties of molecules obtained from a well-defined algorithm or experimental procedure. The OD, ID, 2D and 3D molecular descriptors were calculated using paDelDescriptor software 2.20 version (14) in addition to quantum chemical descriptors generated by the Spartan 14 software.

\section{Data pre-treatment}

Data pre-treatment for the generated molecular descriptors after normalization was done by using "Data pretreatment GUI 1.2" software that uses V-WSP algorithm (15) to remove noise and redundant data. This helps to overcome productivity and generalization failure of the model due to constant value and highly correlated descriptors in forming QSAR models.

\section{Data normalization and Descriptors Transformation}

Molecular descriptors values were normalized by employing " normalized data 1.0 version software " to give each variable the same opportunity and make the relationship between descriptors considerably less demanding. The molecular descriptors of the training set were transformed through normalization (16) using the mathematical equation below:

$$
x^{n}=\frac{X-X_{\max }}{X_{\max }-X_{\min }}
$$

Where $X^{n}$ is the normalized descriptor, $X_{\max }$ is the maximum value in a descriptor column and $X_{\min }$ is the minimum value in the column of the training dataset.

\section{Assessing Quality Assurance of the Model}

Statistical parameters of the model were reviewed and evaluated to ascertained its fitting ability, reliability, predictive ability, stability and robustness of the model generated. The quality assurance of a developed model is guaranteed if the following parameters are satisfied; $R^{2}$ pred $>0.5$, $\mathrm{Q}^{2}>0.6, P(95 \%)<0.05$, high value of F-test, low values of $R^{2}$ random and $Q^{2}$ random.

\section{Validation of the Model}

Leave-one-out cross validation technique was employed to determine the predictive power of the model. This was evaluated by using this mathematical expression:

$$
Q_{c v}^{2}=1-\left[\frac{\sum\left(Y_{\text {pred }}-Y_{\text {exp }}\right)^{2}}{\sum\left(Y_{\text {exp }}-Y_{\text {training }}^{-}\right)}\right]
$$

Where $Y_{\text {pred, }} Y_{\exp }$ and $\bar{Y}_{\text {training symbolized the }}$ experimental, the predicted and mean values of experimental activity of training set compounds.

Also, the square of the correlation coefficient for the test set $\left(R^{2}\right.$ test $)$ was evaluate for the predictive capacity of the developed model as part of the external validation technique. The closer the value of $R^{2}$ test value to 1.0 the better the model. The $R^{2}$ test is evaluated by using this mathematical equation:

$$
R_{\text {test }}^{2}=1-\frac{\sum\left(Y_{\text {pred }}-Y_{\text {test }}\right)^{2}}{\sum\left(Y_{\text {pred }}-Y_{\text {training }}\right)^{2}}
$$

Where $Y_{\text {pred }}$ and $Y_{\text {test }}$ are the predicted and experimental activity values of the test set compounds. $\bar{Y}_{\text {training }}$ is the mean (average) activity value of the training set.

\section{Y - Randomization test}

$Y$ - randomization is an important external validation technique to ascertained that a developed QSAR model is strong and reliable and is not inferred by luck. Y-randomization test is performed on the training dataset. The low values of $\mathrm{R}^{2}$ and $\mathrm{Q}^{2}$ is an indication that the model is very robust and highly reliable, and the ${ }_{C} R^{2}{ }_{p}$ value of the model must be greater than 0.5 to pass the $\mathrm{Y}$-randomization test. The ${ }_{C} R^{2}{ }_{\mathrm{P}}$ value is calculated by using this mathematical formula:

$$
c R_{p}^{2}=R \times\left[R^{2}-\left(R_{r}\right)^{2}\right]^{2}
$$

Where

${ }_{c} R^{2}{ }_{p}=$ coefficient of determination for $\mathrm{Y}$ Randomization 
$\mathrm{R}=$ Coefficient of correlation for $\mathrm{Y}$-Randomization $R_{r}=$ Average " $R$ " of random models.

\section{Degree of contribution of selected descriptors}

The level of contribution of each descriptor in the model is determined by calculating its standardized regression coefficients $b j$ using this mathematical equation:

$$
b_{j}=\frac{s_{j} b_{j}}{S Y}=J=1, \ldots . . d
$$

Where $b_{j}$ is the regression coefficient of descriptor $j . S_{j}$ and $S_{y}$ are the standard deviations for each descriptor and activity respectively.

The descriptor of higher absolute standardized coefficient implies a greater importance to the rest of molecular descriptors.

\section{Multi-co-linearity evaluation}

Multi-co-linearity estimation among descriptors selected by GFA analysis is evaluated using variance inflation factor (VIF) by the mathematical expression below:

$$
V I F_{i}=\frac{1}{1-R_{i j}^{2}}
$$

Where $R^{2}{ }_{i j}$ is the correlation coefficient of the multiple regression between the descriptor $\mathrm{i}$ and the rest $\mathrm{j}$ descriptors in the developed model (17).

\section{Assessment of the applicability domain of the model}

Evaluation of the applicability domain of a model is a significant step to confirm that the developed model is capable to make a reliable prediction within the chemical space for which it was developed (16). To describe the applicability domain of the QSAR model, the leverage approach was employed.

Leverage of a given dataset hi, is defined by this mathematical expression:

$$
H_{i}=x_{i}\left(X^{T} X\right)^{-1} X_{i}^{T}
$$

Where $x i$ the descriptor row is vector of the considered compound $i$, hi is the $\mathrm{n} \times \mathrm{k}$ descriptor matrix of the training set compound used to generate the model.

The warning leverage $\left(h^{*}\right)$ is the limit of normal values of $x$ outliers and is expressed mathematically as:

$$
h^{*}=\frac{3(p+1)}{n}
$$

Where $\mathrm{n}=$ number of training compounds and $\mathrm{P}$ is the number of predictor variables (descriptors) in the model.

If the leverages $\mathrm{hi}<\mathrm{h}^{*}$ for the test compounds, it considered to be reliably predicted by the developed model.

The relevance area of the model in terms of chemical space is visualized by the plot of standardized residuals against leverage values (Williams plot).

\section{MOLECULAR DOCKING SIMULATION}

The molecular interactions studies were carried out on a Dell computer system, with processor

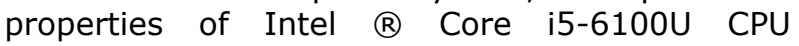
Dual@2.30GHz, 12 GB (RAM) between the ligands and two neurotransmitter transporters (targets); the Crystal structure of LEUTAA, a bacterial homolog of $\mathrm{Na}+/ \mathrm{Cl}$--dependent neurotransmitter transporters and X-ray structure of dopamine transporter elucidates antidepressant mechanism as to elucidate which of the NET inhibitors will have the best binding affinity against any of these two receptors, because the current structural findings of human monoamine neurotransmitters transporters (MATs) is based on X-ray crystal structures of bacterial and invertebrate homologs (18).

\section{Making of Ligand and Target}

All the compounds were optimized using Spartan software initially saved as SDF files and were appropriately later saved as Protein Data Bank (PDB) files. Subsequently, crystal structure of LEUTAA, a bacterial homolog of $\mathrm{Na}+/ \mathrm{Cl}--$ dependent neurotransmitter transporters and Xray structure of dopamine transporter elucidates antidepressant mechanism (targets) were downloaded from Protein Data Bank website with PDB codes 2 A65 and 4M48 respectively. Fig.1 below displays the prepared structure of the receptors. 


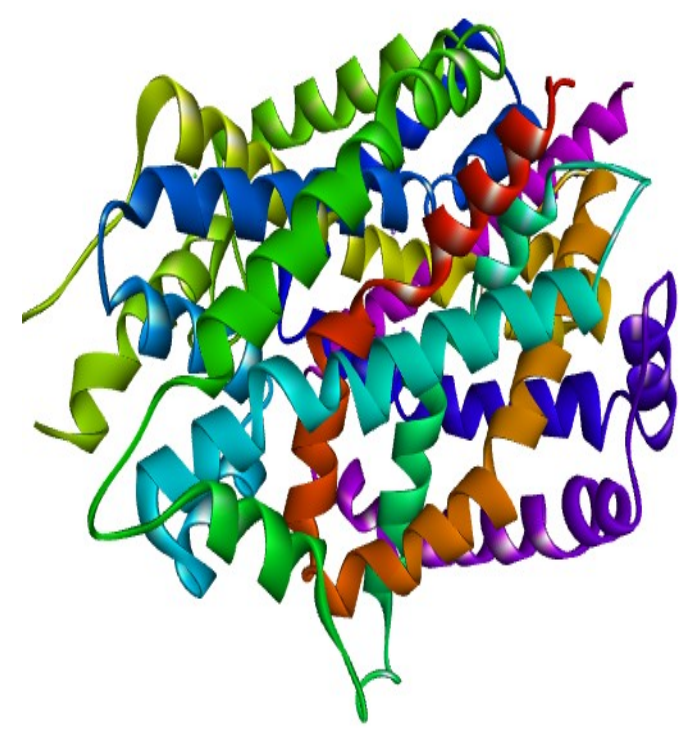

(a) PDB Code $2 \mathrm{~A} 65(1.65 \AA)$

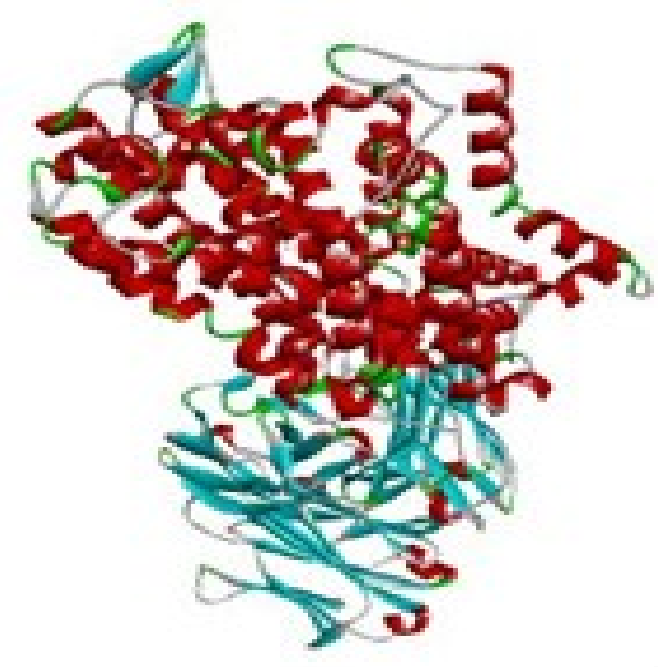

(b) PDB Code 4 M48 ( $2.99 \AA)$

Figure 1: Prepared structures of the targets.

\section{Docking process}

The docking of the prepared ligands with the receptors $2 A 65$ and $4 M 48$ were conducted using the AutoDock Vina version 4.0 of Pyrex software. Hence, Discovery Studio software was used in visualizing the molecular interactions of the stable complex.

\section{RESULTS AND DISCUSSIONS}

QSAR study was explored to investigate the structure-activity relationship of 50 compounds with distinguishing organic fragments acting as norepinephrine transporter (NET) inhibitors. The nature of models in a QSAR study is expressed by its fitting the data points through regression and making predictions of isolated dataset.

\section{QSAR on pKi of norepinephrine transporter (NET) inhibitors}

A data set of 50 compounds was divided into a training set of 36 compounds used in developing the model and a test set of 14 compounds was used to evaluate the predictive ability of the QSAR model for the inhibition of norepinephrine transporter. The predicted and experimental activities alongside with their residual values were presented in Supplementary Table S1. The low residual values resulted from the experimental and predicted activities is a good indication that the developed model has good predictability.

The descriptive statistics parameters for the training set and test set activities value were reported in Table 2. Comparison the descriptive statistics parameters between training and test set in Table 2, the values for the training set were approximately equal to that of test set. This shows that the test set is interpolative within the training set, and the similarity in the activity distribution of training set and test set. This is a good quality assurance that Kennard Stone's algorithm used in this research generates a test set that is a true reflection of the training set.

The genetic algorithm-multiple linear regression (GA-MLR) examination prompted the choice of 6 descriptors, which were eventually used to amassed a linear regression model for calculating pKi of norepinephrine transporter inhibitors within the chemical space of the model. The model with statistical significance was selected and represented by equation (10) below: 


$$
\begin{aligned}
& p K I=2.788(\boldsymbol{A L o g} \boldsymbol{P})+3.382(\boldsymbol{A A T S} 7 \boldsymbol{i})+3.782(\boldsymbol{A T S C} 3 \boldsymbol{p})+2.234(\boldsymbol{I C 2})-5.147(\text { GGI10 }) \\
& +3.728(\text { RDF75u })+0.989 \\
& \mathrm{~N}_{\text {train }}=36, \mathrm{R}_{\text {train }}=0.9156, \mathrm{R}_{\text {adjusted }}^{2}=0.8982, \mathrm{Q}^{2} \text { Loo }=0.8755, \text { Outliers }>3.0=0 \mathrm{~N}_{\text {test }}=14, \mathrm{R}_{\text {test }}^{2}= \\
& 0.5832
\end{aligned}
$$

$\mathrm{N}$ is the total number of the datasets, $\mathrm{R}^{2}$ is the squared correlation coefficient, $\mathrm{Q}^{2}$ Loo is the squared cross-validation coefficients for leave one out. In the model, the number of ratio of training set data to the ratio number of descriptors present in the model was 6 and in agreement with Topliss ratio (19). This implies that the developed model obeyed the QSAR semi-empirical rule of thumb (20). The name and the symbol of the descriptors, the standardized regression coefficients (degree of contribution) and percentage contribution of the descriptors were reported in Table 5. The combined presence of $2 \mathrm{D}$ and $3 \mathrm{D}$ descriptors in the developed model is an evidence that these types of descriptors are able to characterize good antipsychotic activity of the compounds. The sign, magnitude and percentage contribution of each descriptor is not only to give critical information on the direction of influence of the descriptor but also pinpoint the strength of contribution to the activity of the compound.

The model generated was subjected to internal and external validations. The outcome of internal and external validations of the model is in conformity to Occam's razor rule. The generally acceptable QSAR Model Validation Tools and the validated parameters of the model were presented in Table 1 . The values of validation parameters of the model were in agreement with generally acceptable QSAR Model Validation Tools

\begin{tabular}{|c|c|c|c|c|}
\hline $\begin{array}{l}\text { Validatio } \\
\text { n Tools } \\
\end{array}$ & Interpretation & $\begin{array}{l}\text { Acceptable } \\
\text { Value }\end{array}$ & $\begin{array}{l}\text { Developed } \\
\text { model } \\
\text { Value }\end{array}$ & Remarks \\
\hline $\mathrm{R}^{2}$ & Co-efficient of determination & $\geq 0.6$ & 0.911 & pass \\
\hline$P(95 \%)$ & Confidence interval at $95 \%$ confidence level & $<0.05$ & 2.446 & pass \\
\hline$Q^{2} c v$ & Cross-Validation Co-efficient & $>0.5$ & 0.870 & pass \\
\hline$R^{2}-Q^{2} c V$ & Difference between $\mathrm{R}^{2}$ and $\mathrm{Q}$ & $\leq 0.3$ & 0.04 & pass \\
\hline $\mathrm{N}_{\text {Ext testset }}$ & Minimum number of external and test sets & $\geq 5$ & 14 & pass \\
\hline$R^{2}$ Testset & $\begin{array}{l}\text { Co-efficient of determination of external and } \\
\text { test set }\end{array}$ & $\geq 0.5$ & 0.5850 & pass \\
\hline $\mathrm{CR}_{\mathrm{p}}^{2}$ & $\begin{array}{l}\text { Coefficient of determination for } Y \text { - } \\
\text { randomization }\end{array}$ & $>0.5$ & 0.840 & pass \\
\hline$R_{\text {adj }}^{2}$ & Adjusted R-squared & $>0.6$ & 0.893 & Pass \\
\hline VIF & Variance Inflation Factor & $<10$ & $1.4-4.4$ & Pass \\
\hline t-test & t-Statistic value & $>2$ & $5-9$ & Pass \\
\hline
\end{tabular}
reported in Table 1 . This confirmed the reliability, stability and robustness of the developed model.

Table 1: Accepted QSAR Model Validation Tools (21).

The Pearson's correlation matrix and other statistical tools employed for validation of the model were reported in Table 3. The low value in correlation coefficients between each pair of descriptors $(<7.0)$ is a clear indication that there was no significant multi-collinearity among the descriptors in the developed model. The Variance
Inflation Factor (VIF) values reported in Table 3 were less than 10 and the t-statistics values were greater than 2 for all the descriptors. This is a quality assurance that the developed model was statistically significant, and the descriptors contributed appreciably to the model at $95 \%$ level (21) and they were orthogonal. 
Olasupo et al. JOTCSA. 2020; 7(1): 179-196.

RESEARCH ARTICLE

Table 2: Descriptive statistical analysis of NET inhibitor compounds

\begin{tabular}{l|ccc}
\hline $\begin{array}{l}\text { Descriptive } \\
\text { values }\end{array}$ & $\begin{array}{l}\text { Training } \\
\text { dataset }\end{array}$ & $\begin{array}{l}\text { Test } \\
\text { dataset }\end{array}$ & \\
\hline Dataset Number & 36 & 14 & \\
Standard Error & 0.185 & 0.296 & \\
Median & 7.054 & 7.497 & \\
Standard & & 1.108 & 1. \\
Deviation & 1.108 & 1.223 & \\
Sample Variance & 1.227 & 2.677 & \\
Kurtosis & -0.632 & -1.264 & \\
Skewness & 0.229 & -436 & \\
Range & 4.439 & 4.436 & \\
Minimum & 5.084 & 4.500 & \\
Maximum & 9.523 & 8.936 & \\
Mean & 6.940 & 7.394 & \\
\hline
\end{tabular}

Table 3: Pearson's correlation matrix and model quality assurance

\begin{tabular}{|c|c|c|c|c|c|c|c|c|c|}
\hline & & & & & & & VIF & $\begin{array}{l}\mathrm{t}- \\
\text { statistics }\end{array}$ & D value \\
\hline & $A \log P$ & AATS7i & ATSC $3 p$ & $I C 2$ & GGI10 & RDF75u & & & \\
\hline ALogP & 1 & & & & & & 1.5021 & 7.5604 & 2.47E08 \\
\hline AATS7i & -0.3321 & 1 & & & & & 1.4789 & 7.4649 & $3.16 \mathrm{E}-08$ \\
\hline ATSC $3 p$ & -0.2592 & -0.2991 & 1 & & & & 1.4376 & 9.4970 & $2.1 \mathrm{E}-10$ \\
\hline IC2 & -0.2742 & 0.0487 & 0.0765 & 1 & & & 1.4177 & 5.8502 & $2.4 \mathrm{E}-06$ \\
\hline GGI10 & -0.2382 & 0.0921 & -0.1711 & 0.5005 & 1 & & 4.5022 & -9.5663 & $1.79 \mathrm{E}-10$ \\
\hline RDF75u & -0.2940 & 0.2215 & -0.1337 & 0.4759 & 0.6377 & 1 & 4.3800 & 6.7912 & 1.87E-07 \\
\hline
\end{tabular}


Olasupo et al. JOTCSA. 2020; 7(1): 179-196.

RESEARCH ARTICLE

Table 4: Y-randomization table for QSAR Analysis

\begin{tabular}{l|lll}
\hline Model & $\mathrm{R}$ & $\mathrm{R}^{\wedge} 2$ & $\mathrm{Q}^{\wedge} 2$ \\
\hline Original & 0.9545 & 0.9111 & 0.8702 \\
Random 1 & 0.4197 & 0.1762 & -0.2759 \\
Random 2 & 0.3402 & 0.1157 & -0.4558 \\
Random 3 & 0.3943 & 0.1555 & -0.3333 \\
Random 4 & 0.4690 & 0.2199 & -0.2220 \\
Random 5 & 0.4408 & 0.1943 & -0.1861 \\
Random 6 & 0.1560 & 0.0243 & -0.6456 \\
Random 7 & 0.3589 & 0.1288 & -0.3166 \\
Random 8 & 0.3237 & 0.1048 & -0.3536 \\
Random 9 & 0.3323 & 0.1104 & -0.4357 \\
Random 10 & 0.3646 & 0.1329 & -0.3307 \\
\hline
\end{tabular}

Random Models Parameters

\begin{tabular}{ll}
\hline Average $r:$ & 0.3599 \\
Average $r^{\wedge} 2:$ & 0.1363 \\
Average $Q^{\wedge} 2:$ & -0.3555 \\
CRp^2: & 0.8439 \\
\hline
\end{tabular}

Table 5: Names of the model descriptors and their respective degree of contribution

\begin{tabular}{|c|c|c|c|c|}
\hline Descriptor & Descriptor Name & Type & $\begin{array}{l}\text { Degree of } \\
\text { contribution }\end{array}$ & $\begin{array}{l}\text { percentage of } \\
\text { contribution }\end{array}$ \\
\hline ALogP & $\begin{array}{l}\text { Ghose-Crippen LogKow } \\
\text { Average Broto-Moreau autocorrelation } \\
\text { - lag } 7 \text { / weighted by first ionization }\end{array}$ & $2 \mathrm{D}$ & 0.513 & 13.3 \\
\hline AATS7i & $\begin{array}{l}\text { potential } \\
\text { Centered Broto-Moreau }\end{array}$ & $2 \mathrm{D}$ & 0.500 & 13.0 \\
\hline ATSC3p & $\begin{array}{l}\text { autocorrelation - lag } 3 \text { / weighted by } \\
\text { polarizabilities }\end{array}$ & $2 \mathrm{D}$ & 0.631 & 16.4 \\
\hline IC2 & $\begin{array}{l}\text { Information content } \quad \text { index } \\
\text { (neighborhood symmetry of 2-order) }\end{array}$ & $2 \mathrm{D}$ & 0.383 & 10.0 \\
\hline GGI10 & Topological charge index of order 10 & $2 \mathrm{D}$ & -1.061 & 27.6 \\
\hline RDF75u & $\begin{array}{l}\text { Radial distribution function - } 075 / \\
\text { unweighted }\end{array}$ & $3 D$ & 0.756 & 19.7 \\
\hline
\end{tabular}

The model generated was used to predict the test set data, and the results were reported in Supplementary Table S1. The predicted pKi values for the training and test sets were plotted against the experimental pKi values as shown in Figure 3. Similarly, the plot of the standardized residuals values for both the training and test sets against the leverage values of the descriptors in the model were shown in Fig. 4. As can be seen from Supplementary Table S1, Figure 3 and Figure 4, the calculated values for the pKi were in excellent agreement with those of the test set, as a result of this, no any form of error was displayed by the model. 


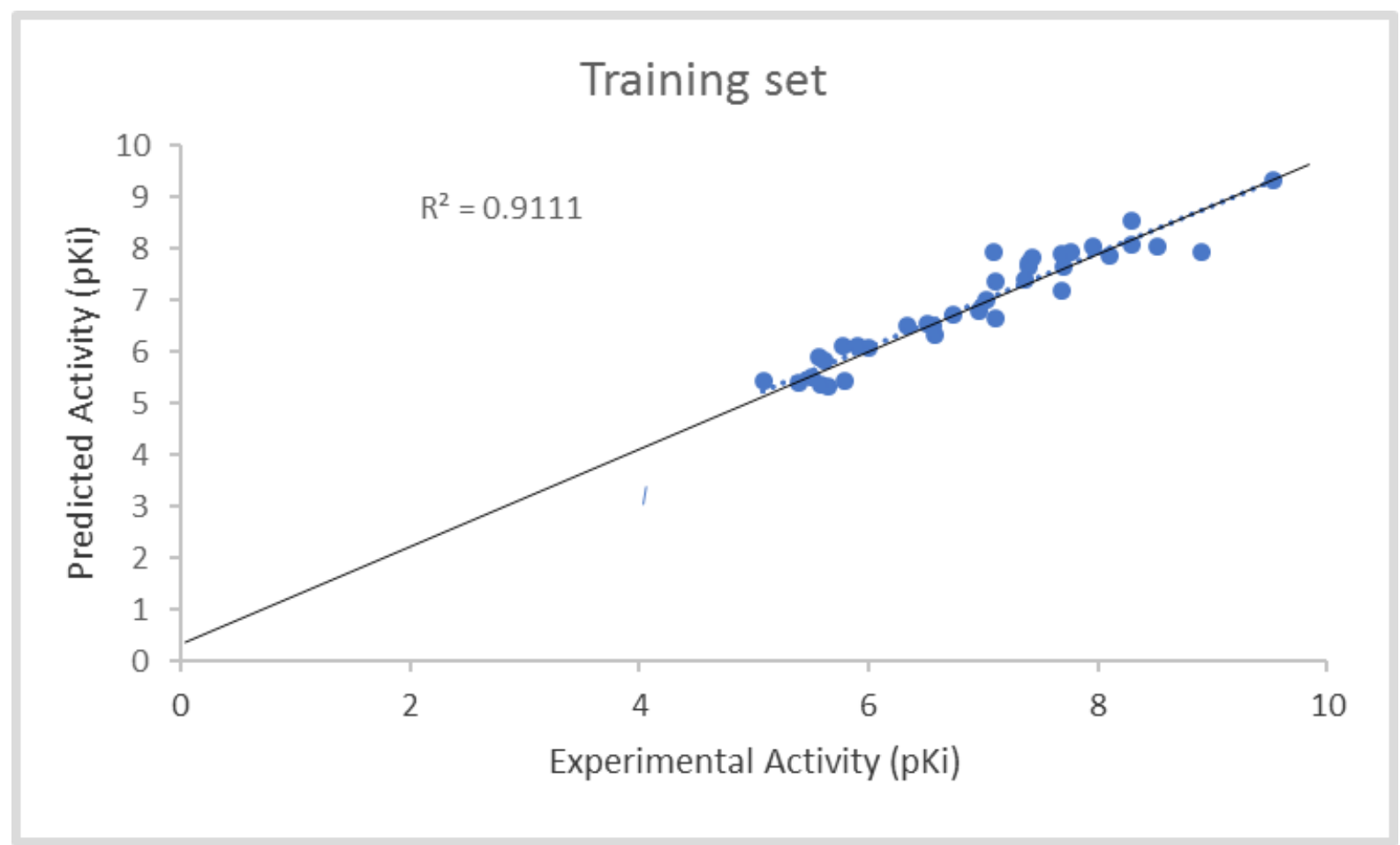

Figure 2: Plot of predicted pKi values against Experimental pKi values for Training.

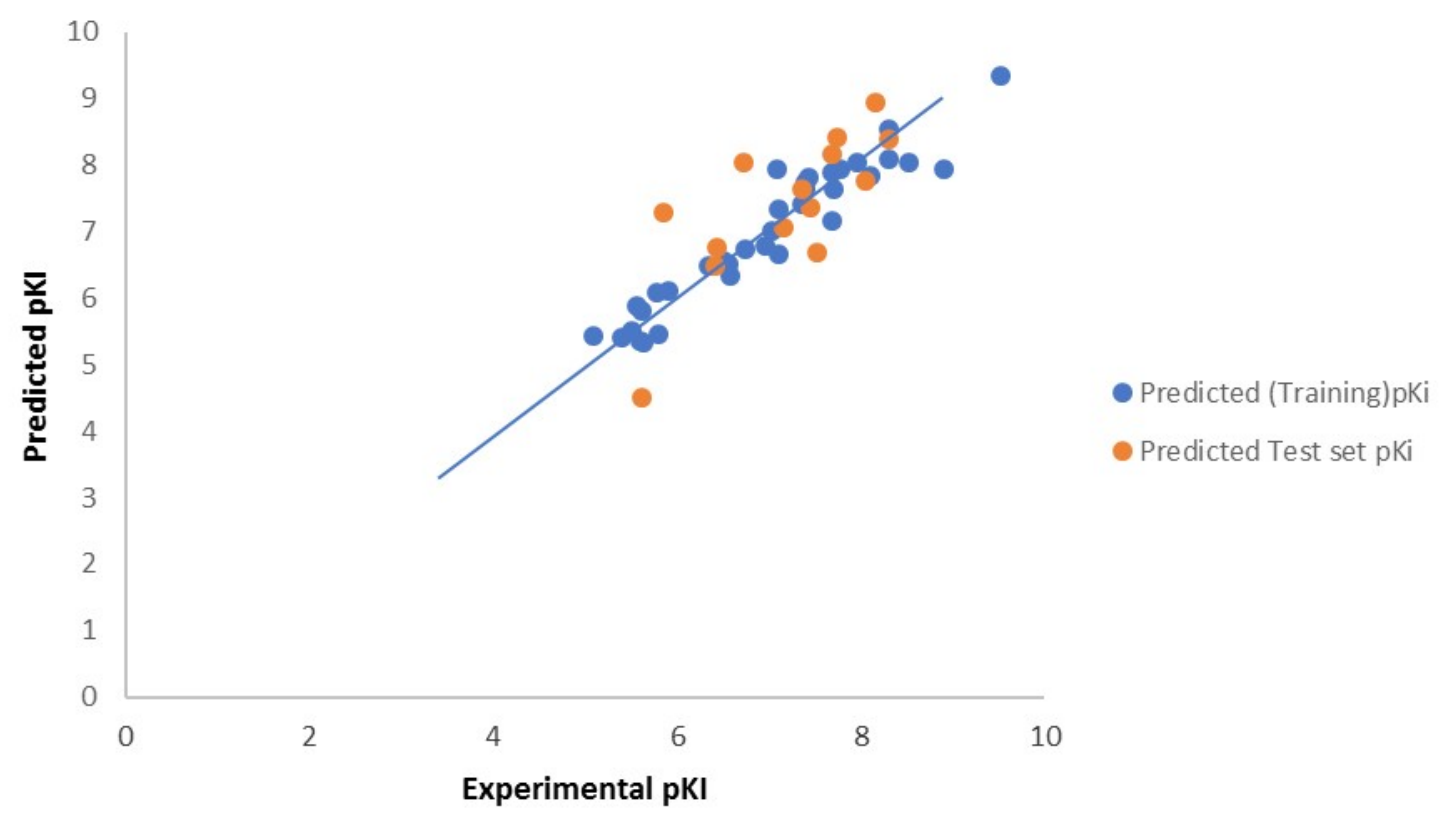

Figure 3: Plot of predicted pKi values against Experimental pKi values for Training and Test sets. 


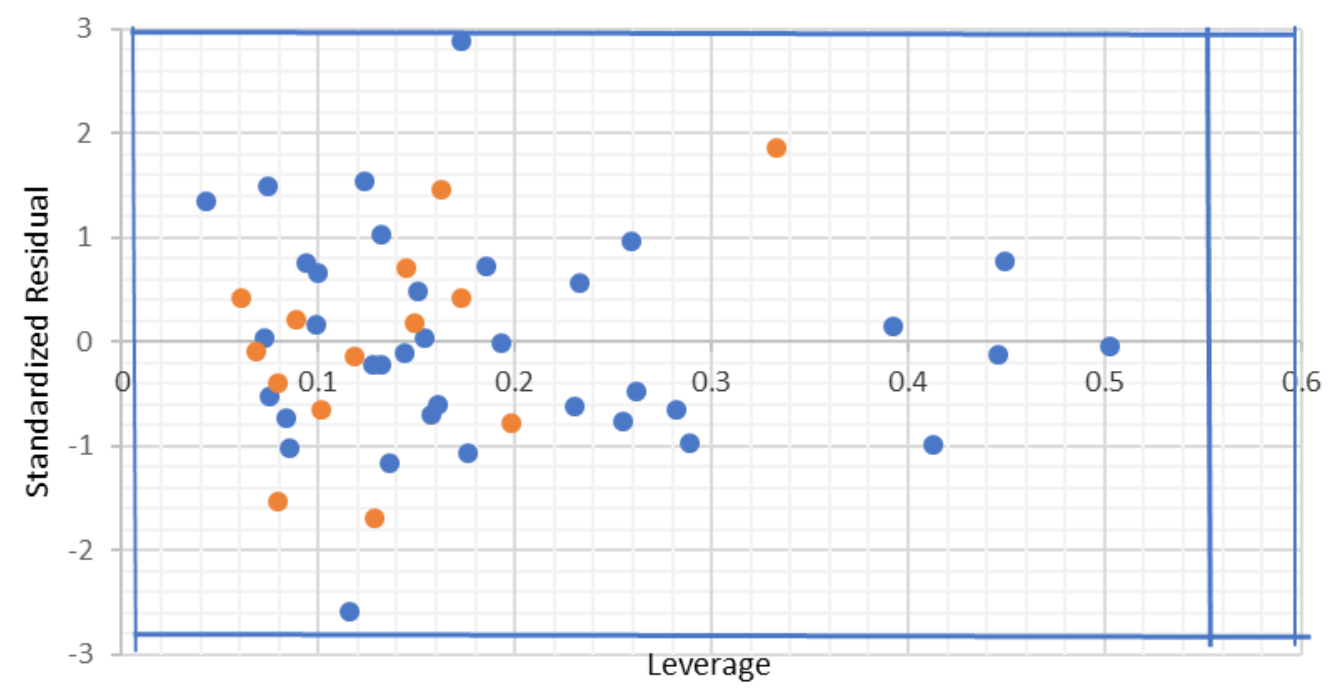

\section{- Train Std Residual - Test Std Residual}

Figure 4: A Williams plot for the data set of pKi standardized residual against its descriptor space.

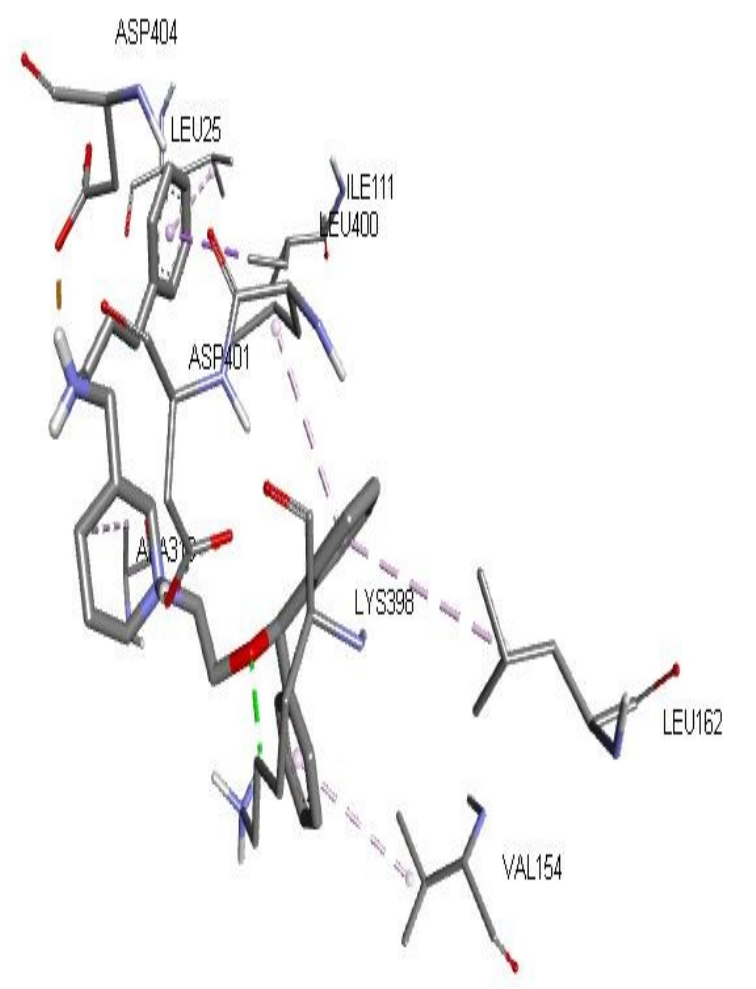

$(12 \mathrm{a} 3 \mathrm{D})$
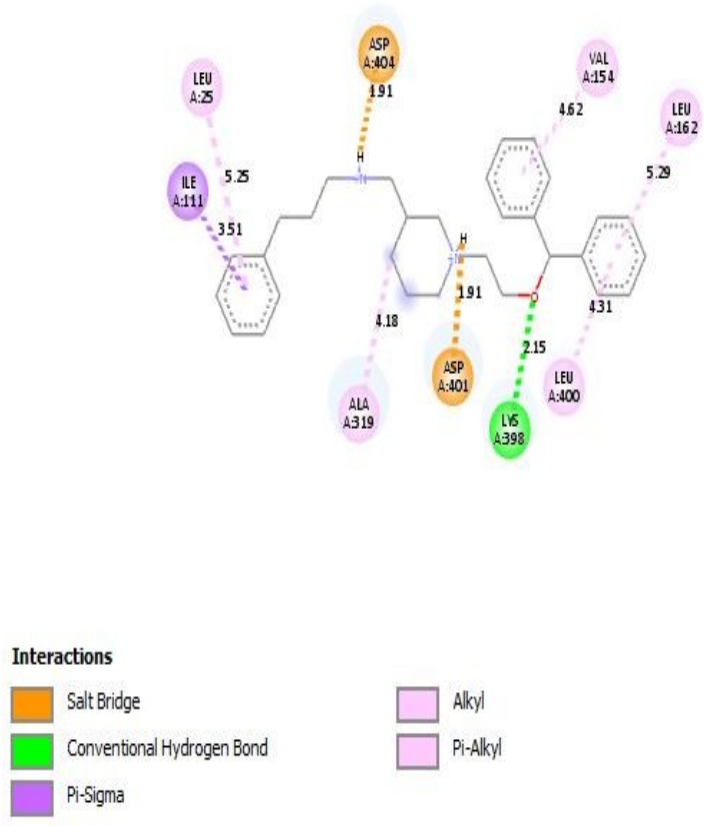

$(12 \mathrm{a} 2 \mathrm{D})$ 


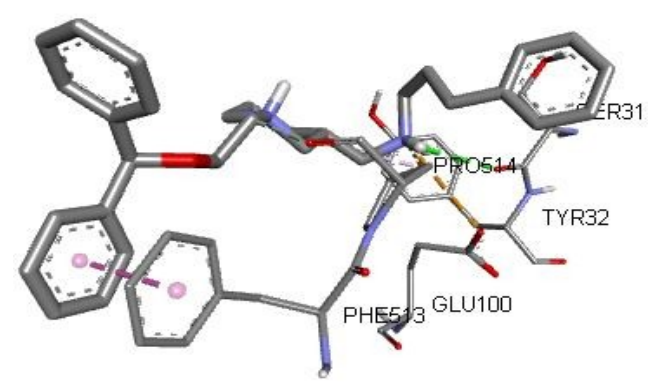

$(12 \mathrm{~m} 3 \mathrm{D})$

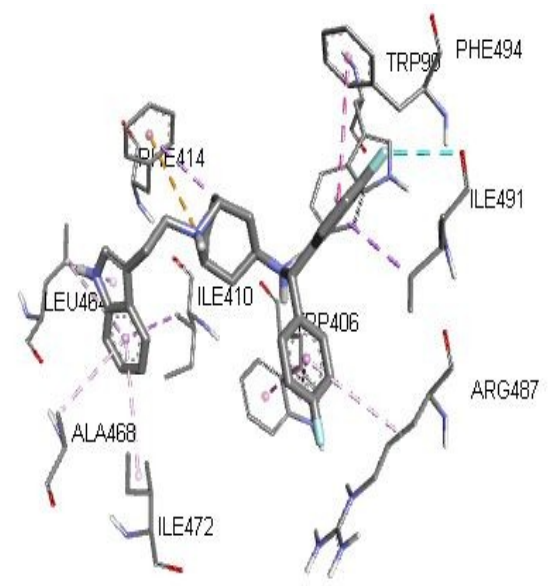

(38a3D)

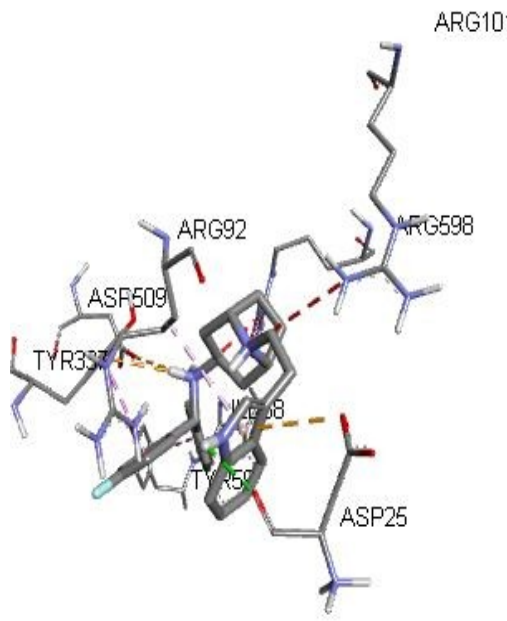

$(38 \mathrm{~m} 3 \mathrm{D})$
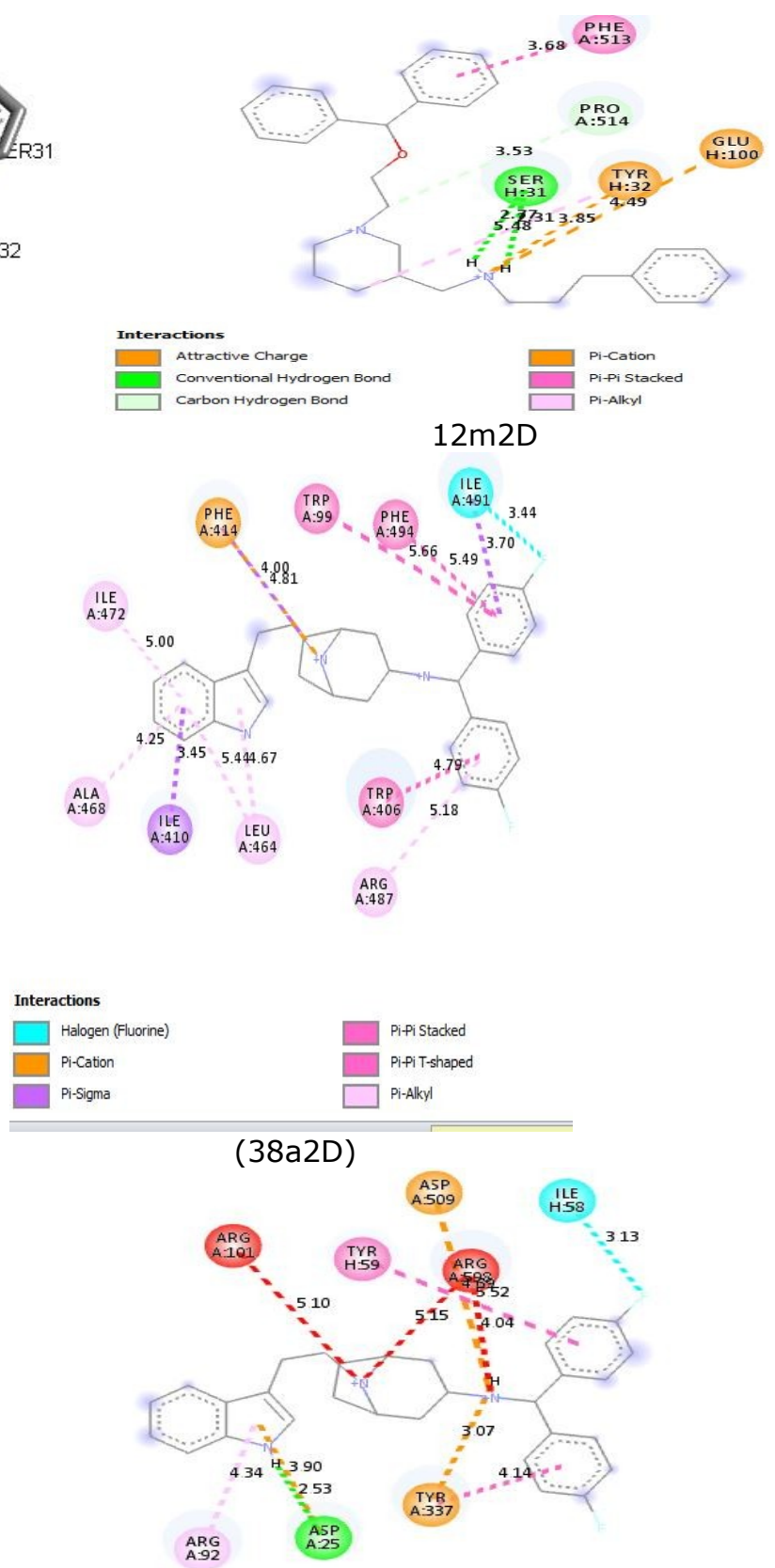

teractions
Attractive Charge
Conventional Hydrogen Bond
Halogen (Fluorine)

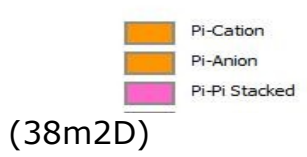




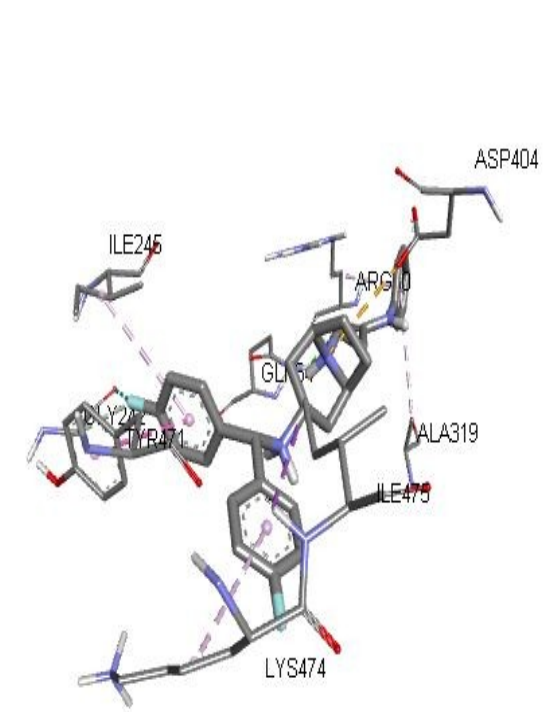

(44a3D)

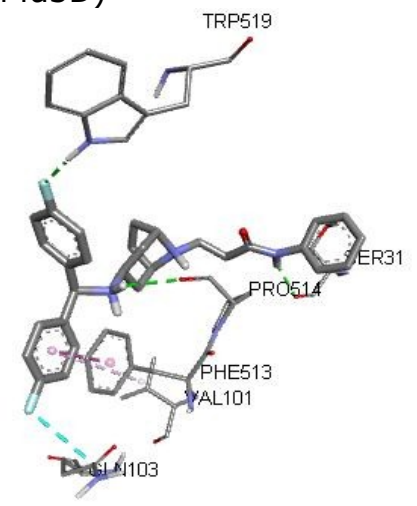

(44m3D)
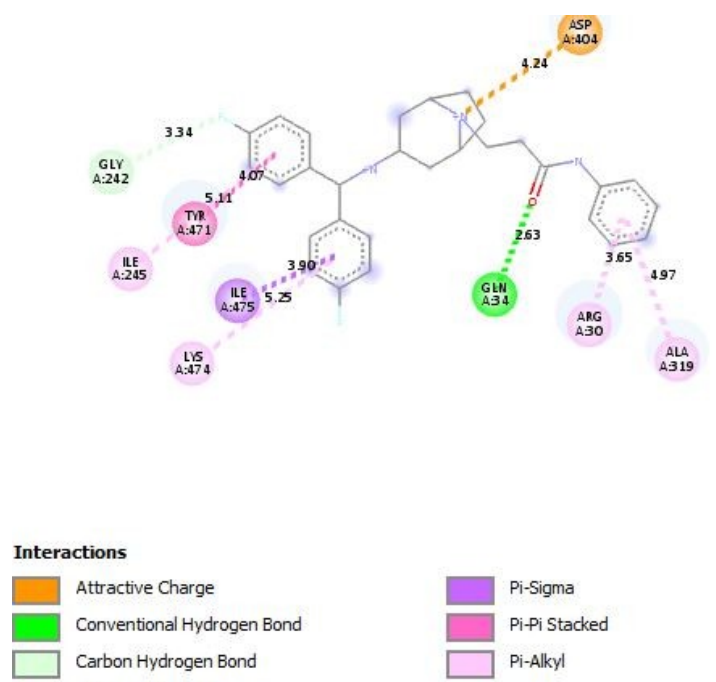

Halogen (Fluorine)
(44a2D)

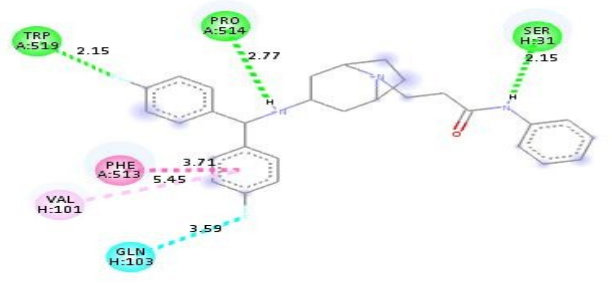

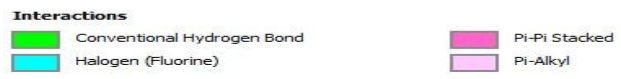

(44m2D)

Figure 5. (12a 2D\&3D), (38a 2D\&3D) and (44a 2D\&3D) depict 2D and 3D interactions at the binding site between receptor PDB code $2 A 65$ with ligand 12, 38 and 44 while (12m 2D\&3D), (38m 2D\&3D) and $(44 \mathrm{~m} 2 \mathrm{D} \& 3 \mathrm{D})$ show $2 \mathrm{D}$ and 3D interactions at the binding site between receptor PDB code $4 \mathrm{M} 48$ with ligand 12, 38 and 44 respectively.

\section{QSAR model validation}

The internal coherence of the training set was established by using leave-one-out crossvalidation technique to ascertained the strength and reliability of the developed model, because the candid significance of a QSAR model is not merely their ability to mimic known activities of chemicals, set by their fitting power $\left(R^{2}\right)$, but above all is their prospective for guessing biological activity accurately. The great value of $\mathrm{Q}^{2}$ Loo for pKi of NET inhibitors used (0.8755) speak well of a fully clad internal validation of the model.

The plot of experimental pKi values against predicted $\mathrm{pKi}$ values for training set was presented in Figure 2. The displayed of linear relationship was observed in the plot between the experimental and predicted activities of the training set $\left(R^{2}=0.911\right)$. The fact that all these results were in agreement with QSAR validation tools presented in Table 1 is a confirmation of the reliability, robustness and stability of the developed model (21).

The Fig. 4, depicts the Williams plot of the NET dataset, in which the standardized residuals for each compound in the dataset were plotted against their leverage values, coming about to acknowledgment of likely outliers and outstanding chemicals in the models.

The applicability domain is set up inside a defined domain where all the data point were within the 
boundary \pm 3 for residuals and a leverage threshold $\mathrm{h} *\left(\mathrm{~h}^{*}=3 \mathrm{p}^{0} / \mathrm{n}\right)$ where $\mathrm{p}^{0}$ is the number of model parameters and $n$ is the number of compounds) (15). Based on our findings, it is clear that every one of the compounds of the training set and test set for the dataset were inside the domain (square area) and no statistical value far from others compounds (outlier) with standardized residuals $>3 d$ for the dataset exist.

The percentage of contribution was calculated to determine the relative importance and the contribution of every descriptor in the model. The degree of contribution of each descriptor and variance inflation factor (VIF) of the descriptor were estimated to evaluate the percentage and the significance of contribution of the descriptors as reported in Table 3 and Table 5 respectively. The descriptor GGI10 showed highest contribution value $(27.6 \%)$ in the model with VIF value of 4.502 as reported in the two tables, but the contribution negatively affects the model as it is observed in the equation (10) with negative regression coefficient.

The robustness and reliability of the model was evaluated through $\mathrm{Y}$-randomization test to ascertain whether the developed model is by chance correlation or not. After few repeated trials to compare the stemmed scores with the scores of the original model with non-randomized data, the new QSAR model generated was observed to have low $\mathrm{R}^{2}$ and $\mathrm{Q}^{2}$ Loo values as reported in Table 4 . The results of this test were clearly in agreement with QSAR validation tools presented in Table 1 . This is an indication that the developed model is robust, good and dynamic. The fact that $\mathrm{CR}^{2}{ }_{\mathrm{p}}$ value $>0.5$, confirms that the model possesses good quality assurance and that the model is not only inferred by chance but also very powerful.

\section{Elucidation of Descriptors in NET pKi model}

By interpreting the molecular descriptors presented in the model (Table 5), it is possible to increase supportive chemical functional groups, fingerprints and pharmacophores into the activities of the NET inhibitors. Therefore, a sufficient interpretation of the QSAR results is given below.

ALogP is a 2D type molecular descriptor, and the first in our QSAR model. It defined as GhoseCrippen LogKow or Ghose-Crippen-Viswanathan octanol-water partition coefficient. (ALogP) is calculated from the AlogP model consisting of a regression equation based on the hydrophobicity contribution of 115 atom types(22)(23). AlogP estimates are provided only for compounds having atoms of types $\mathrm{C}, \mathrm{H}, \mathrm{O}, \mathrm{N}, \mathrm{S}, \mathrm{Se}, \mathrm{P}, \mathrm{B}, \mathrm{Si}$, and halogens.

Each atom in every structure is classified into one of the 115 atom types. Then, estimated logP for any compound is given by:

$$
A \log P=\sum_{i} n_{i} a_{i}
$$

where $\mathrm{n}$ is the number of atom of type $i$ and $\mathrm{a}_{i}$ is the corresponding hydrophobicity constant. The list of the atom types with the corresponding hydrophobicity contributions is given under the list of atom-centered fragments. This descriptor tells us the higher the number of hetero atoms in a molecule, the higher the tendency for this molecule to be less hydrophobic. Since the percentage contribution of the descriptor in this model is $13 \%$, it indicates that more than $10 \%$ of the bioactivity of a lead compound will improve should the number of heteroatoms present be increased.

AATS7i and ATSC3p are defined as Average Broto-Moreau autocorrelation - lag 7 / weighted by first ionization potential and Centered BrotoMoreau autocorrelation - lag 3 / weighted by polarizabilities respectively. They are both 2D autocorrelation descriptors and their respective percentage contribution to the models are given as 13 and $16.4 \%$ respectively in Table 2 . The ATS descriptor describes how a property is distributed along the topological structure. It is a spatial autocorrelation on a molecular graph, which can be used to improve the activity of the compounds by altering the ionization potential and polarizability of the compounds. Since these molecular descriptors contributed positively to the model the pKi values of the compounds can be improved by adding fragments to the compounds that can increase the polarity of the compounds thereby creating the charge stability of the ligands' interaction with the binding sites. GGI10 is a topological charge descriptor defined as Topological charge index of order 10. GGI10 gave the highest contribution in the model, but since its contribution negatively affect the model, then the steady reduction in this descriptor value can improve the Ki values of the dataset. The ability of topological charge indices to describe molecular charge distribution has been 
established by correlating them with the dipole moment of a heterogeneous set of hydrocarbons, and so reducing the number of heterogeneous hydrocarbons presently correlated with the dipole moment of the molecule will lead to an increase in the bioactivity of the compounds.

IC2 is defined in Table 2 as Information content index (neighborhood symmetry of 2-order), it is a 2D type information content descriptor. It gave the least contribution to the model, but $10 \%$ contribution can be significant depending on the nature of the molecule. The IC2 molecular descriptor suggests that by introducing other bonds at that carbon, the structural complexity of the molecules will be increased and the Shannon entropy will also be increased thereby easily activating the interactions of the molecule with the binding site.

RDF75u is an RDF descriptor (Radial Distribution Function descriptors), this descriptor is based on the distance distribution in the geometrical representation of a molecule and constitute a radial distribution function code (RDF code) that shows certain characteristics in common with the 3D-MORSE code. The radial distribution function in this form meets all the requirements for a 3D descriptor, it also provides further valuable information such as bond distances, ring types, planar and non-planar systems. This fact is a most valuable consideration for a computerassisted code elucidation(24). The positive regression coefficient of this descriptor in the model as contained equation (7) with the highest value of degree of contribution as reported in Table 5 is a good indication of its influential contribution to the antipsychotic activity with variation in the bond distance and ring types of the studied compounds.

\section{Docking results}

The docking result of this study is presented in terms of binding affinity ( $\mathrm{kcal} / \mathrm{mol}$ ) as reported in Supplementary Table S1. All the ligands were docked into the active site of the receptors, the Crystal structure of LEUTAA, a bacterial homolog of $\mathrm{Na}+/ \mathrm{Cl}$--dependent neurotransmitter transporters and X-ray structure of dopamine transporter elucidates antidepressant mechanism in order to evaluate their abilities to inhibit these neurotransmitters. The current available findings of human neurotransmitters transporters are based on X-ray crystal structures of bacterial and invertebrate homologs which includes the bacterial amino acid transporters LeuT (PDB:
2A65) and the Drosophila melanogaster (PDB: 4M48) (18) as employed in this study.

The binding affinity values of the two receptors (PDB: $4 M 48$ and PDB: 2A65) for all the studied compounds ranged from $4.4 \mathrm{kcal} / \mathrm{mol}$ to 10.3 $\mathrm{kcal} / \mathrm{mol}$ and were reported in Supplementary Table S1. Ligands 8, 12,26, and 38 had higher binding affinity with the receptor PDB 4 M48 and Ligands 9, 10,12,38 and 44 had higher binding affinity with the receptor PDB 2A65 respectively. The Discovery Studio Visualizer was used to visualize and analyze the three ligands of higher binding affinity that were found to display higher binding affinity and common to the two receptors as shown in Fig. 5.

The binding affinity, hydrogen bond, hydrophobic and electrostatic interactions of the three ligands having higher binding affinity with the two receptors were reported in Table 6 . The number $12 a, 38 a \& 44 a$ represent the interactions of the Ligands (compound 12, 38 and 44 ) between the receptor (PDB ID 2A65) while $12 \mathrm{~m}, 38 \mathrm{~m} \& 44 \mathrm{~m}$ depict the interaction of the ligands (compound 12,38 and 44 ) between the receptor (PDB ID 4 M48) respectively.

All the three ligands (compound 12, 38 and 44) with the higher binding affinity were observed to inhibit the targets by forming hydrogen bonds and hydrophobic interactions with amino acids of the two receptors (PDB ID 2A65) and (PDB ID $4 \mathrm{M} 48$ ) respectively except compound 38 that could not form hydrogen bond with the receptor (PDB ID 2A65) as reported in Table 6. This may inform the higher resolution $(2.99 \AA)$ of the receptor (PDB ID 4M48) compare to the lower resolution (1.65 $\AA$ ) of the other receptor (PDB ID 2A65) (https ://www. rcsb.org). The three ligands were found to be firmly bonded with hydrogen bonds of the receptor (PDB ID 4M48) pocket amino acids (SER31, ASP25, PRO514 and TRP519). The higher number of hydrogen bonds were observed in the two ligands (compound 12 and 44) with the target pockets of the receptor (PDB ID 4M48) which might be connected to their higher activity (compound $12, \mathrm{pKi}=7.383$ and compound 44, pKi $=5.607$ ) contrast with to the other ligand (compound 38, pKi $=5.084$ ) with the lowest activity which formed just a single hydrogen bond with the receptor. This infers a direct relationship between the binding affinity and inhibitory activity of the studied compounds proved from the number of hydrogen bonds formed between the ligands and the receptor. 
However, high binding affinity is evident in the ligand 38 and this might be because of its large number of hydrophobic interactions and electrostatic effect due to the presence of fluorine atom, Pi- Cation, Pi- Sigma, Pi-Pi- stacked, Pi-PiT-shaped, Pi-Alkyl with amino acid residues (ILE491,ILE410, PHE494,ARG487,LEU464. ALA464,ILE472).

\section{CONCLUSIONS}

The QSAR investigation was successfully performed on dataset of 50 norepinephrine transporter (NET) inhibitors, mined from CHEMBL database. The result of the QSAR modelling was reliable because it satisfied the OECD criteria set for a model development. The combination of 2D and 3D descriptors generate a good model to predict the inhibitory activity of the studied compounds. The internal validation reported in the work as $\mathrm{Q}_{2} \mathrm{CV}$ was estimated to 0.870 , while the external validation reported as $\mathrm{R}^{2}$ Pred was given to be 0.583 . This is an excellent indication of a good predictive ability of the model.

The result of Applicability Domain (AD) shows that all the studied compounds were within the defined domain. Molecular docking study were carried out on all the compounds using two neurotransmitter transporters (receptors) PDB IDs $2 A 65$ and $4 M 48$ respectively. Three ligands (compound number 12,38 and 44) showed higher binding affinity were found to best inhibit the two receptors by forming strong hydrogen bonds and hydrophobic interactions with amino acids of the targets. However, higher number of hydrogen bonds were observed between the receptor (PDB ID 4M48) and two ligands (compound 12 and 44) out of the three ligands with higher activity, compound 12 ( $\mathrm{pKi}=7.387$ ) and compound 44
$(\mathrm{pKi}=5.607)$ compare to compound 38 with the lowest activity $(\mathrm{pKi}=5.084)$. This suggests a good correlation between the binding affinity and inhibitory activity of the ligands and that the mechanisms or mode of action of the ligands could be a direct interaction with the receptor (PDB ID 4M48) of higher resolution value (2.99 $\AA)$. Therefore, the two ligands, compound 12, [1(2-(benzhydryloxy)ethyl)-3-(((3phenylpropyl)ammonio)methyl)piperidin-1-ium] and compound 44 [3-((bis(4fluorophenyl)methyl)ammonio)-8-(3-oxo-3(phenylamino)propyl)-8-azabicyclo[3.2.1]octan8-ium] proved to be the most promising hit compounds and the receptor PDB ID 4 M48 (2.99 A) shows to be a better receptor for this investigation which could be linked to its higher resolution value.

The information derived from the QSAR investigation and molecular docking analysis of this study could find a robust application in pharmaceutical industries to design novel NET inhibitors with more potent and more specific therapeutic anti-psychotic agents.

\section{FUNDING}

The Authors received no direct funding for this study.

\section{ACKNOWLEDGEMENTS}

We wish to acknowledge members of theoretical and physical chemistry group, chemistry department, Ahmadu Bello University Zaria. We sincerely appreciate David Arthur and Abdulfatai Usman for their technical support and advice in the course of this study. 
Table 6. Molecular interactions between the three ligands of higher binding affinity and the two receptors.

\begin{tabular}{|c|c|c|c|c|c|c|}
\hline $\begin{array}{l}\text { Ligand } \\
\text { ID }\end{array}$ & $\begin{array}{l}\text { Ligand } \\
\text { Number }\end{array}$ & $\begin{array}{l}\text { Binding } \\
\text { Affinity } \\
\text { (kcal/mol) }\end{array}$ & $\begin{array}{l}\text { Hydrogen } \\
\text { bond }\end{array}$ & & Hydrophobic interactions & $\begin{array}{l}\text { Electrostatics } \\
\text { Interactions }\end{array}$ \\
\hline & & & Amino acid & Bond length $(\AA)$ & Amino Acid & Amino Acid \\
\hline \multirow[t]{2}{*}{ CHEMBL67078 } & $12 a$ & -9.3 & LYS398 & 2.15279 & $\begin{array}{l}\text { ILE111, ALA319, VAL154, LEU162, LEU400, } \\
\text { LEU25 }\end{array}$ & \\
\hline & $12 \mathrm{~m}$ & -7.35 & SER31 & 2.76717 & PHE513,TYR32 & TYR32 \\
\hline \multirow[t]{2}{*}{ CHEMBL197384 } & $38 a$ & -10.3 & SER31 & 2.31044 & $\begin{array}{l}\text { ILE491, } \quad \text { ILE410, } \\
\text { PHE494,ARG487,LEU464.ALA464,ILE472 }\end{array}$ & PHE414 \\
\hline & $38 \mathrm{~m}$ & -7.5 & ASP25 & 2.53334 & TYR337, TYR59, ARG92 & ASP25 \\
\hline \multirow[t]{3}{*}{ CHEMBL200310 } & $44 a$ & -9.9 & GLN34 & 2.62533 & $\begin{array}{l}\text { TLE475, } \\
\text { LYS474,ARG30,ALA319 }\end{array}$ & ASP404 \\
\hline & $44 m$ & -8.45 & PRO514 & 2.15327 & PHE513, VAL101 & \\
\hline & & & $\begin{array}{l}\text { SER31 } \\
\text { TRP519 }\end{array}$ & $\begin{array}{l}2.76554 \\
2.1523\end{array}$ & & \\
\hline
\end{tabular}




\section{REFERENCES}

1. Zhou J. NIH Public Access. 2006;29(12):1235-44.

2. Rice DP, Miller LS. Health economics and cost implications of anxiety and other mental disorders in the United States. Br J Psychiatry. 1998;173(S34):4-9.

3. Xue W, Yang F, Wang $P$, Zheng $G$, Chen $Y$, Yao $X$, et al. What Contributes to SerotoninNorepinephrine Reuptake Inhibitors' DualTargeting Mechanism? The Key Role of Transmembrane Domain 6 in Human Serotonin and Norepinephrine Transporters Revealed by Molecular Dynamics Simulation. ACS Chem Neurosci. 2018;9(5):1128-40.

4. Blakely RD, Bauman AL. Biogenic amine transporters: regulation in flux. Curr Opin Neurobiol. 2000;10(3):328-36.

5. Deorah S, Lynch CF, Sibenaller ZA, Ryken TC. Trends in brain cancer incidence and survival in the United States: Surveillance, Epidemiology, and End Results Program, 1973 to 2001. Neurosurg Focus. 2006;20(4):E1.

6. Penmatsa A, Wang KH, Gouaux E. X-ray structure of dopamine transporter elucidates antidepressant mechanism. Nature [Internet]. 2013;503(7474):85-90. Available from: http://dx.doi.org/10.1038/nature12533

7. Dessalew N. QSAR study on dual SET and NET reuptake inhibitors: an insight into the structural requirement for antidepressant activity. J Enzyme Inhib Med Chem. 2009;24(1):262-71.

8. Om A-S, Ryu J-C, Kim J-H. Quantitative structure-activity relationships for radical scavenging activities of flavonoid compounds by GA-MLR technique. Mol Cell Toxicol. 2008;4(2):170-6.

9. Davis GDJ, Vasanthi AHR. QSAR based docking studies of marine algal anticancer compounds as inhibitors of protein kinase $B$ (PKBß). Eur J Pharm Sci. 2015;76:110-8.

10. Hehre WJ, Huang WW. Chemistry with computation: an introduction to SPARTAN. Wavefunction, Inc.; 1995.
11. Bauernschmitt R, Ahlrichs R. Treatment of electronic excitations within the adiabatic approximation of time dependent density functional theory. Chem Phys Lett. 1996;256(45):454-64.

12. Kennard RW, Stone LA. Computer aided design of experiments. Technometrics. 1969;11(1):137-48.

13. Zeng $H$, Zheng $R$, Guo $Y$, Zhang $S$, Zou $X$, Wang $N$, et al. Cancer survival in $C$ hina, 2003-2005: A population-based study. Int J cancer. 2015;136(8):1921-30.

14. Yap CW. PaDEL-descriptor: An open source software to calculate molecular descriptors and fingerprints. J Comput Chem. 2011;32(7):1466-74.

15. Roy K, Kar S, Ambure P. On a simple approach for determining applicability domain of QSAR models. Chemom Intell Lab Syst. 2015;145:22-9.

16. Tropsha A, Gramatica P, Gombar VK. The importance of being earnest: validation is the absolute essential for successful application and interpretation of QSPR models. QSAR Comb Sci. 2003;22(1):69-77.

17. Arthur DE, Uzairu A, Mamza P, Abechi SE, Shallangwa G. Qsar Modelling of Some Anticancer Pgi 50 Activity on HI - 60 Cell Lines. 2016;3(1).

18. Andersen J, Ringsted $\mathrm{KB}$, Bang-Andersen $B$, Strømgaard K, Kristensen AS. Binding site residues control inhibitor selectivity in the human norepinephrine transporter but not in the human dopamine transporter. Sci Rep. 2015;5:15650.

19. Topliss JG, Edwards RP. Chance factors in studies of quantitative structure-activity relationships. J Med Chem. 1979;22(10):123844.

20. Damme $S$ Van, Bultinck P. A new computer program for QSAR-analysis: ARTEQSAR. J Comput Chem. 2007;28(11):1924-8.

21. Tropsha A. Best practices for QSAR model development, validation, and exploitation. Mol Inform. $2010 ; 29(6-7): 476-88$.

22. Viswanadhan VN, Ghose AK, Revankar GR, Robins RK. Atomic physicochemical 
parameters for three dimensional structure directed quantitative structure-activity relationships. 4. Additional parameters for hydrophobic and dispersive interactions and their application for an automated superposition of certain . J Chem Inf Comput Sci. $1989 ; 29(3): 163-72$.

23. Ghose AK, Crippen GM. Atomic physicochemical parameters for three- dimensional structure-directed quantitative structure-activity relationships I. Partition coefficients as a measure of hydrophobicity. J Comput Chem. 1986;7(4):565-77.

24. Caballero J, Fernández M. Artificial neural

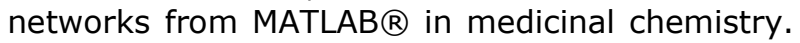
Bayesian-regularized genetic neural networks (BRGNN): Application to the prediction of the antagonistic activity against human platelet thrombin receptor (PAR-1). Curr Top Med Chem. 2008;8(18): 1580-605. 